\title{
Intentional Forgetting: An Emerging Field in Al and Beyond
}

\author{
Christoph Beierle ${ }^{1} \cdot$ Ingo J. Timm ${ }^{2}$
}

Published online: 11 December 2018

○ Gesellschaft für Informatik e.V. and Springer-Verlag GmbH Germany, part of Springer Nature 2018

\section{Why do Humans Forget?}

In human evolution, learning has evolved as a key strategy for adaptation. While animals adapt on the basis of their own experiences, humans integrate complex forms of interaction and information exchange in the learning process to include other people's experiences in addition to stimulus-responseconditioning. By lifting parts of the adaptation to the cognitive level, humans consciously reason about the learning process, i.e., they continuously perceive new information and extend their knowledge by learning. Due to limited cognitive capacity, new information is not just added but irrelevant information is sorted out, e.g., we know what we have had for breakfast today but fade out breakfast details of the last days. We have established memory structures enabeling us to remember or derive knowledge fragments with considerable success, albeit possibly requiring also considerable effort, even when we have forgotten about them. Human learning and forgetting is not just steered by subcognitive routines, as in the example of remembering breakfast, but seems also to rely on a memory organization supporting to intentionally deliberate on previously or implicitly known information fragments.

In current research both in psychology and information systems, intentionally balancing learning and forgetting is of great interest to meet information overload as a new challenge in the information or digital society. Current trends, like the digital transformation and ubiquitous computing, cause a massive increase in available data and information. However, the problem of information overload does not only

Christoph Beierle

beierle@fernuni-hagen.de

Ingo J. Timm

itimm@uni-trier.de

1 Faculty of Mathematics and Computer Sciences, FernUniversität in Hagen, 58084 Hagen, Germany

2 Center for Informatics Research and Technology (CIRT), Trier University, 54296 Trier, Germany tackle human but also computer systems being bounded by physical memory. From a complexity perspective, many (if not most) AI algorithms exhibit exponential runtime or space complexity. As a result, even slight increases in the amount of information available may have a disproportionate effect on the runtime or memory requirements for those algorithms.

Therefore, the need for forgetting in computer science has already been recognized in academics and even in politics. From a normative perspective, the question arises whether there is a right to be forgotten, e.g., whether it is mandatory to provide mechanisms in the Internet or online social networks, such that data or information that has become available once can be removed or forgotten later on [6]. From a technological perspective, this interpretation of forgetting addresses technical mechanisms for deleting or reordering data and information. While this is an inherent functionality of any computer system, in cloud and cluster computing it remains a severe challenge as for an individual living in todays digital epoch keeping control of the specific physical location of data becomes practically infeasible.

\section{Should AI Systems Forget?}

Especially in AI systems, a sophisticated concept of forgetting is required, as knowledge and the acquisition of knowledge is ubiquitous: "Although additional knowledge can enhance the performance of a problem solver by reducing its requirements, it can also have exactly the opposite effect. In particular, additional knowledge can have the effect of greatly increasing search time [...]" [9, p. 116].

For knowledge-based systems or in the context of semantic technologies, there is a dominance of logic-based representation of knowledge. To implement such systems, operators for adding new knowledge, e.g., facts or rules, are provided. Depending on the specific logic, there are prerequisites to be considered when manipulating the knowledge base, e.g., consistency. Forgetting has also been addressed in logics; for instance, forgetting operators have been proposed 
for propositional, first-order, as well as description logics, e.g., $[4,8,13]$. These aim at eliminating variables, terms, or facts from the explicitly or implicitly available knowledge without changing what is independent of the forgotten elements [1]. Consequently, these operators are important prerequisites for pruning knowledge bases to cope with computational complexity and for revising beliefs in dynamically changing environments [2].

Acquiring new knowledge by algorithms is in the focus of machine learning. Various machine learning approaches aggregate information or abstract input data for a better generalization [7]. In context of forgetting, machine learning can serve as an implicit forgetting function because, e.g., irrelevant attributes or features are faded out. The concept of forgetting can also improve machine learning capabilities. For instance, Nakayama and Yoshii [10] proposed a data selection approach with passive (fading out old data) and active (deciding not to consider obstacle data) forgetting for artificial neural network training.

The consideration of forgetting is not limited to a single knowledge base. In distributed AI, knowledge is distributed across several agents for preserving natural distributivity of the real-world as well as for reducing complexity [3, 5]. Key features of such systems are communication and coordination enabling social deliberation in multiagent systems. Thus, reasoning and planning problems are transfered into coordination tasks among agents with limited individual knowledge [11]. Furthermore, knowledge distributions can be adapted dynamically at runtime. On the individual level, approaches from learning and logics are relevant to implement forgetting features.

Obviously, there are many other important research fields in AI where different kinds and facets of forgetting play an important role, e.g., robotics, planning, or subsymbolic machine learning. The use of forgetting functions within AI systems is also of great concern if real-world applications are in question.

Due to the dramatic changes in handling of information within our society, the theoretical and methodological approaches indicated above provide a good starting point, but are often limited with respect to volume, velocity, and variety of (Big) data. AI systems based on huge data and information sources are facing potential information overload, such that autonomous capabilities to balance learning and forgetting are required along with adequate transparency. As humans also use forgetting as an intentional process - as considered in psychology - the question arises whether AI systems can benefit from explicitly activating forgetting operators.

Recently, the German national science foundation (Deutsche Forschungsgemeinschaft) initiated the new interdisciplinary priority research program "Intentional Forgetting in Organizations" (DFG-SPP 1921), currently funding eight interdisciplinary projects in the areas of psychology, computer science, and business information systems. This initiative as well as the research directions and challenges outlined above underline the urgent need of investigating intentional forgetting, and there are already strong indications of its high potential. Indeed, intentional forgetting must be viewed as an emergent research field for AI and beyond [12]. The objective of this special issue is to present recent work on intentional forgetting from an $\mathrm{AI}$ research perspective.

\section{Contributions: Approaches to (Intentional) Forgetting}

In the field of knowledge representation and reasoning, forgetting has not received as much attention as other processes like query answering, inference, or update. However, from a cognitive view, forgetting also has an ordering function in the human mind, suppressing information that is deemed irrelevant and improving cognitive capabilities to focus and deal only with relevant aspects of the problem under consideration. In their article A Brief Survey on Forgetting from a Knowledge Representation and Reasoning Perspective, Thomas Eiter and Gabriele Kern-Isberner collect and survey approaches to forgetting in the field of knowledge representation and reasoning. The paper recalls forgetting operations for propositional and predicate logic, as well as for answer set programming and modal logics, discusses forgetting in the context of (ir)relevance and (in)dependence, and elaborates on the positive impact of forgetting on solving problems of knowledge representation and reasoning.

In the second technical contribution of this special issue, Please Delete That! Why Should I? Explaining learned irrelevance classifications of digital objects, Michael Siebers and Ute Schmid report on the assistive system Dare2Del. The cognitive companion system Dare2Del is intended to support users managing digital objects in a working environment and to facilitate intentional forgetting of irrelevant digital objects. The core of Dare2Del is an interpretable machine learning mechanism which induces decision rules to classify whether a digital objects is irrelevant. Siebers and Schmid formalize the decision making process as logic inference, present a method to generate verbal explanations for irrelevance decisions, and point out how such explanations can be constructed on different levels of details.

Knowledge workers already face an overwhelming mass of information in their daily life, and trends like digital transformation even intensify this phenomenon. In Managed Forgetting to Support Information Management and Knowledge Work, Christian Jilek, Yannick Runge, Claudia Niederée, Heiko Maus, Tobias Tempel, Andreas Dengel, and Christian Frings investigate knowledge work and 
information management support measures inspired by human forgetting to address this problem. They report on experiences gained with the prototype of a forgetful information system used in their daily work for the last three years, pointing out first solutions as well as challenges that still need to be tackled.

The technically defined notions of forgetting in knowledge representation often differ substantially from the common-sense understanding of forgetting. In Towards a General Framework for Kinds of Forgetting in CommonSense Belief Management, Christoph Beierle, Gabriele Kern-Isberner, Kai Sauerwald, Tanja Bock, and Marco Ragni elaborate and identify kinds and contexts of forgetting from a common-sense perspective. The authors present an abstract formalization of operations involving forgetting and instantiate and refine this abstract framework with conditional beliefs and ordinal conditional functions, also covering forgetting operations inspired by cognitive psychology.

In times of Big Data and Industry 4.0, organizational information as well as knowledge availability and quantity are driving complex decision-making tasks. By transferring theories from human team cognition to $\mathrm{AI}$ systems, the AdaptPRO project adopts intentional forgetting as a strategy for coping with information overload in both human and multiagent teams. The article Intentional Forgetting in Distributed Artificial Intelligence by Lukas Reuter, Jan Ole Berndt, Anna-Sophie Ulfert, Conny Antoni, Thomas Ellwart, and Ingo J. Timm gives an overview of the interdisciplinary research project AdaptPRO, focusing on knowledge distributions and knowledge dynamics from a distributed AI perspective. Its core contribution is a formal model for distributing and adapting (meta-)knowledge by intentional forgetting to enable efficient and resilient teamwork.

In their discussion paper Psychological Perspectives on Intentional Forgetting. An Overview of Concepts and Literature, Thomas Ellwart and Annette Kluge argue that psychological models of forgetting offer numerous theoretical perspectives that can be transferred to AI research and practice. This applies especially for the development of socio-digital systems with artificial intelligence where the question arises how to deal with growing amounts of information. The article presents a short terminological overview and systematization of psychological concepts of forgetting from an individual, group, and organizational level.

We are happy that two well-known researchers shared their views on forgetting and intentional forgetting with us in an interview. Norbert Gronau, Professor for Business Informatics, Processes and Systems at the University of Potsdam, sees a huge potential for organizations in this field. Otthein Herzog, Professor at the Jacobs University Bremen, at the University of Bremen, and at Tongji University, Shanghai, PRC, argues that intentional forgetting must be an integral part of any system dealing with dynamic knowledge.
This special issue is complemented by abstracts of two recent doctoral dissertations both dealing with conditional knowledge bases and the inference relations they induce, which is a core topic in knowledge representation and reasoning. Christian Eichhorn addresses rational reasoning methods for qualitative conditionals of the form If $A$, then usually $B$ and their semantics given by ranking functions. Marc Finthammer studies concepts and algorithms for reasoning with quantitative conditionals of the form If A holds, then $B$ holds with probability $p$ under the principle of maximum entropy.

We are grateful to many persons for their effort in making this special issue possible. In particular, we thank all authors for their hard work in preparing their articles, and all referees for their time and expertise in carefully reviewing the contributions. We are also indebted to the KI Editorial Board for their willingness to dedicate a special issue to the emerging field of intentional forgetting, and to the whole KI team for their support in organizing this special issue.

\section{Content}

\subsection{Technical Contributions}

- A Brief Survey on Forgetting from a Knowledge Representation and Reasoning Perspective

Thomas Eiter and Gabriele Kern-Isberner

- Please Delete That! Why Should I? Explaining learned irrelevance classifications of digital objects

Michael Siebers and Ute Schmid

- Managed Forgetting to Support Information Management and Knowledge Work

Christian Jilek, Yannick Runge, Claudia Niederée, Heiko Maus, Tobias Tempel, Andreas Dengel, and Christian Frings

- Towards a General Framework for Kinds of Forgetting in Common-Sense Belief Management

Christoph Beierle, Gabriele Kern-Isberner, Kai Sauerwald, Tanja Bock, and Marco Ragni

\subsection{Project Reports}

- Intentional Forgetting in Distributed Artificial Intelligence

Lukas Reuter, Jan Ole Berndt, Anna-Sophie Ulfert, Conny Antoni, Thomas Ellwart, and Ingo J. Timm

\subsection{Discussion}

- Psychological Perspectives on Intentional Forgetting. An Overview of Concepts and Literature

Thomas Ellwart and Annette Kluge 


\subsection{Interviews}

- Intentional Forgetting-A Huge Potential for Organizations · Interview with Prof. Norbert Gronau, University of Potsdam

- Intentional Forgetting Must be Part of the Functionality - Interview with Prof. Otthein Herzog, Jacobs University Bremen, University of Bremen, and Tongij University, Shanghai

\subsection{Doctoral Dissertations}

- Qualitative Rational Reasoning with Finite Conditional Knowledge Bases. Theoretical and Implementational Aspects

Christian Eichhorn

- Concepts and Algorithms for Computing Maximum Entropy Distributions for Knowledge Bases with Relational Probabilistic Conditionals

Marc Finthammer

\section{Service}

\subsection{Conferences and Workshops}

- International Joint Conference on Artificial Intelligence (IJCAI)

- International Conference on the Principles of Knowledge Representation and Reasoning (KR)

- International Joint Conference on Autonomous Agents and Multi-Agent Systems (AAMAS)

- European Conference on Artificial Intelligence (ECAI)

- European Conference on Logics in Artificial Intelligence (JELIA)

- International Conference on Agents and Artificial Intelligence (ICAART)

- German Conference on Artificial Intelligence (KI)

- Biannual conference of the German Society for Cognitive Science (KogWis)

- Annual Conference of the Cognitive Science Society (CogSci)

\subsection{Journals}

- Artificial Intelligence http://www.journals.elsevier.com/ artificial-intelligence

- Journal of Artificial Intelligence Research http://jair.org

- Journal of Applied Logic http://www.journals.elsevier. com/journal-of-applied-logic
- International Journal on Cognitive Science https://www. journals.elsevier.com/cognition

\subsection{Projects}

- ForgetIT project funded by the EC within the 7th Framework Programme https://www.forgetit-project.eu/en/ home/

- DFG Priority Programm SPP 1921 Intentional Forgetting in Organizations http://www.spp1921.de/

\section{References}

1. Ackermann W (1935) Untersuchungen über das eliminationsproblem der mathematischen logik. Math Ann 110(1):390-413

2. Baral C, Zhang Y (2005) Knowledge updates: semantics and complexity issues. Artif Intell 164(1):209-243

3. Bond AH, Gasser L (eds) (1988) Readings in distributed artificial intelligence. Morgan Kaufmann Publ, San Mateo

4. Delgrande JP (2017) A knowledge level account of forgetting. J Artif Intell Res 60:1165-1213

5. Durfee EH, Rosenschein JS (1994) Distributed problem solving and multi-agent systems: comparisons and examples. In: Distributed Artificial Intelligence, Technical Report WS-94-02, pp 52-62. AAAI Press

6. Koops BJ (2011) Forgetting footprints, shunning shadows: a critical analysis of the "right to be forgotten" in big data practice. SCRIPTed 8(3):229-256. https://doi.org/10.2139/ssrn.1986719

7. Kubat M, Bratko I, Michalski RS (1998) A review of machine learning methods. In: Michalski RS, Bratko I, Kubat M (eds) Machine learning and data mining: methods and applications. Wiley, Chichester, pp 3-69

8. Lin F, Reiter R (1994) Forget it! In: Proc. of the AAAI Fall Symposium on Relevance, pp 154-159. AAAI Press, Menlo Park, CA

9. Markovitch S, Scott PD (1993) Information filtering: selection mechanisms in learning systems. Mach Learn 10(2):113-151

10. Nakayama H, Yoshii K (2002) Effectiveness of active forgetting in machine learning applied to financial problems. J Telecommun Inf Technol 3:24-29

11. Ossowski S (2008) Coordination and agreement in multi-agent systems. In: Klusch M, Pechoucek M, Polleres A (eds) Cooperative Information Agents XII, 12th International Workshop, CIA 2008, LNCS, vol 5180. Springer, Berlin, pp 16-23

12. Timm IJ, Staab S, Siebers M, Schon C, Schmid U, Sauerwald K, Reuter L, Ragni M, Niederée C, Maus H, Kern-Isberner G, Jilek C, Friemann P, Eiter T, Dengel A, Dames H, Bock T, Berndt JO, Beierle C (2018) Intentional forgetting in artificial intelligence systems: Perspectives and challenges. In: Trollmann F, Turhan A (eds) KI 2018: advances in Artificial Intelligence, LNCS, vol 11117, pp 357-365. Springer

13. Wang Z, Wang K, Topor R, Pan JZ (2010) Forgetting for knowledge bases in DL-Lite. Ann Math Artif Intell 58(1):117-151 